\title{
Distributions of zeros of solutions to first order delay dynamic equations
}

\author{
D O'Regan', SH Saker², AM Elshenhab² and RP Agarwal ${ }^{3 *}$
}

\begin{tabular}{l}
\hline${ }^{*}$ Correspondence: \\
Agarwal@tamuk.edu \\
${ }^{3}$ Department of Mathematics, Texas \\
A\&M University, Kingsvilie, TX 78363, \\
USA \\
Full list of author information is \\
available at the end of the article
\end{tabular}

available at the end of the article

\begin{abstract}
This paper is concerned with the distributions of zeros of solutions to first order delay dynamic equations on time scales. The results are obtained using iterative sequences.

MSC: 26A15; 26D10; 26D15; 39A13; 34A40; 34N05

Keywords: distribution of zeros; delay equations; time scales
\end{abstract}

\section{Introduction}

The oscillation and distributions of zeros of solutions of first order delay differential and difference equations are studied widely in the literature; see [1-18] and the references therein. However, there are only a few papers considering the distribution of zeros of solutions of first order delay and advanced dynamic equations on time scales (see $[8,19])$. In [12], Zhou considered the first order delay differential equation

$$
x^{\prime}(t)+p(t) x(t-\tau)=0, \quad \text { for } t \in\left[t_{0}, \infty\right),
$$

where

$$
\int_{t-\tau}^{t} p(s) d s \geq \rho>\frac{1}{e}, \quad \text { and } \quad \rho<1,
$$

and established lower and upper bounds for the quotient $x(t-\tau) / x(t)$. In particular the author proved that $x(t-\tau) / x(t) \geq f_{n}(\rho)$ and $x(t-\tau) / x(t)<g_{m}(\rho)$, where the sequences $f_{n}(\rho)$ and $g_{n}(\rho)$ are defined by

$$
\left\{\begin{array}{lll}
f_{1}(\rho)=e^{\rho}, & f_{n+1}(\rho)=e^{\rho f_{n}(\rho)}, & n=1,2, \ldots \\
g_{1}(\rho)=\frac{2(1-\rho)}{\rho^{2}}, & g_{m+1}(\rho)=\frac{2(1-\rho) g_{m}^{2}(\rho)}{g_{m}^{2}(\rho) \rho^{2}+2}, & m=1,2, \ldots
\end{array}\right.
$$

and using these sequences the author studied the distribution of zeros of solutions of (1.1). In [13], Zhang and Zhou considered the first order delay differential equation

$$
x^{\prime}(t)+p(t) x(\tau(t))=0, \quad \text { for } t \in\left[t_{0}, \infty\right),
$$

(c) The Author(s) 2017. This article is distributed under the terms of the Creative Commons Attribution 4.0 International License (http://creativecommons.org/licenses/by/4.0/), which permits unrestricted use, distribution, and reproduction in any medium, provided you give appropriate credit to the original author(s) and the source, provide a link to the Creative Commons license, and indicate if changes were made. 
and studied the distribution of zeros of solutions using the two sequences $f_{n}(\rho)$ and $g_{m}(\rho)$ where

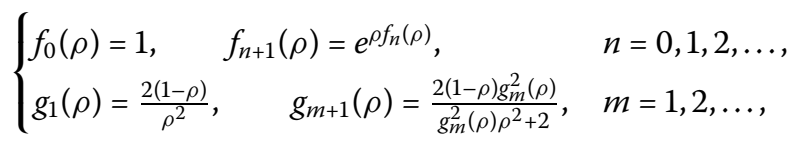

and

$$
\int_{\tau(t)}^{t} p(s) d s \geq \rho>0, \quad \text { and } \quad 0<\rho<1
$$

Zhang and Lian in [19] initiated the study of the distribution of zeros of dynamic equations on time scales and in particular, they considered the first order delay dynamic equation

$$
x^{\Delta}(t)+p(t) x(\tau(t))=0, \quad \text { for } t \in\left[t_{0}, \infty\right)_{\mathbb{T}},
$$

on a time scale $\mathbb{T}$, where $p \in \mathbf{C}_{\mathrm{rd}}\left(\mathbb{T}, \mathbb{R}^{+}\right)$is a non-negative rd-continuous function, $\tau \in$ $\mathbf{C}_{\mathrm{rd}}(\mathbb{T}, \mathbb{T})$ is strictly increasing, $\tau(t)<t$ for $t \in \mathbb{T}$ and $\lim _{t \rightarrow \infty} \tau(t)=\infty$. In [19] the authors established lower and the upper bounds for the quotient $x(\tau(t)) / x(t)$ using the sequences $f_{n}$ and $g_{m}$ where

$$
f_{0}(\rho)=1, \quad f_{n}(\rho)=e^{(1-\rho) f_{n-1}(\rho)}, \quad n=1,2, \ldots
$$

and

$$
\left\{\begin{array}{l}
g_{1}(\rho)=\frac{2 \rho}{(1-\rho)^{2}-2 M(1-\rho)}, \\
g_{m}(\rho)=\frac{2 \rho}{(1-\rho)^{2}-2 M(1-\rho)+\frac{2}{g_{m-1}^{2}(\rho)}}, \quad m=2,3, \ldots
\end{array}\right.
$$

and where $M<(1-\rho) / 2$ and $0 \leq \rho<1$ satisfies the condition

$$
\sup _{\lambda \in E}\left\{\lambda \exp \left\{\int_{\tau(t)}^{t} \zeta_{\mu(s)}(-\lambda p(s)) \Delta s\right\}\right\} \leq \rho
$$

where $E=\{\lambda: \lambda>0,1-\lambda p(t) \mu(t)>0\} ; \zeta_{\mu(s)}$ and $\mu(s)$ will be defined later.

Motivated by these papers, we study the distribution of zeros of oscillatory solutions of the delay dynamic equation (1.5) on a time scale $\mathbb{T}$ by considering new sequences $f_{n}$ and $g_{m}$. In the next section, we present some basic ideas on time scales. In Section 3, we establish lower and upper bounds for $x(\tau(t)) / x(t)$ and in Section 4 , we study the distribution of zeros of solutions of (1.5).

\section{Some preliminaries and lemmas}

In this section, we present some preliminaries; see [20, 21]. A time scale $\mathbb{T}$ is an arbitrary nonempty closed subset of the real numbers $\mathbb{R}$. The forward and backward jump operators are defined by

$$
\sigma(t):=\inf \{s \in \mathbb{T}: s>t\}, \quad \text { and } \quad \rho(t):=\sup \{s \in \mathbb{T}: s<t\}
$$


with $\inf \emptyset=\sup \mathbb{T}$ and $\sup \emptyset=\inf \mathbb{T}$. The graininess function $\mu$ on a time scale $\mathbb{T}$ is defined by $\mu(t):=\sigma(t)-t$. For a function $f: \mathbb{T} \rightarrow \mathbb{R}$ the (delta) derivative is defined by

$$
f^{\Delta}(t)=\frac{f(\sigma(t))-f(t)}{\sigma(t)-t}
$$

if $f$ is continuous at $t$ and $t$ is right-scattered. If $t$ is not right-scattered then the derivative is defined by

$$
f^{\Delta}(t)=\lim _{s \rightarrow t} \frac{f(t)-f(s)}{t-s},
$$

provided this limit exists. A function $f$ is said to be $\Delta$-differentiable if its $\Delta$-derivative exists. A useful formula is $f^{\sigma}=f(\sigma(t))=f(t)+\mu(t) f^{\Delta}(t)$. We will make use of the following product and quotient rules for the derivative of the product $f g$ and the quotient $f / g$ (where $g g^{\sigma} \neq 0$, and here $g^{\sigma}=g \circ \sigma$ ) of two $\Delta$-differentiable functions $f$ and $g$ :

$$
(f g)^{\Delta}=f^{\Delta} g+f^{\sigma} g^{\Delta}=f g^{\Delta}+f^{\Delta} g^{\sigma}, \quad\left(\frac{f}{g}\right)^{\Delta}=\frac{f^{\Delta} g-f g^{\Delta}}{g g^{\sigma}} .
$$

Let $f: \mathbb{R} \rightarrow \mathbb{R}$ be continuously differentiable and suppose $g: \mathbb{T} \rightarrow \mathbb{R}$ is delta differentiable. Then $f \circ g: \mathbb{T} \rightarrow \mathbb{R}$ is delta differentiable and the chain rule,

$$
(f \circ g)^{\Delta}(t)=\left\{\int_{0}^{1} f^{\prime}\left(g(t)+h \mu(t) g^{\Delta}(t)\right) d h\right\} g^{\Delta}(t),
$$

holds. A special case of (2.1) is

$$
\left[x^{\lambda}(t)\right]^{\Delta}=\lambda \int_{0}^{1}\left[h x^{\sigma}+(1-h) x\right]^{\lambda-1} x^{\Delta}(t) d h .
$$

For $s, t \in \mathbb{T}$, a function $F: \mathbb{T} \rightarrow \mathbb{R}$ is called an antiderivative of $f: \mathbb{T} \rightarrow \mathbb{R}$ provided $F^{\Delta}=$ $f(t)$ holds for all $t \in \mathbb{T}$. In this case we define the integral of $f$ by $\int_{s}^{t} f(\tau) \Delta \tau=F(t)-F(s)$. For $a, b \in \mathbb{T}$, and a $\Delta$-differentiable function $f$, the Cauchy integral of $f^{\Delta}$ is defined by $\int_{a}^{b} f^{\Delta}(\tau) \Delta \tau=f(b)-f(a)$. The integration by parts formula reads

$$
\int_{a}^{b} f(t) g^{\Delta}(t) \Delta t=[f(t) g(t)]_{a}^{b}-\int_{a}^{b} f^{\Delta}(t) g^{\sigma}(t) \Delta t
$$

and infinite integrals are defined as

$$
\int_{a}^{\infty} f(t) \Delta t=\lim _{b \rightarrow \infty} \int_{a}^{b} f(t) \Delta t
$$

A function $p: \mathbb{T} \rightarrow \mathbb{R}$ is called regressive if $1+\mu(t) p(t) \neq 0$ for $t \in \mathbb{T}$. A function $p: \mathbb{T} \rightarrow \mathbb{R}$ is called positively regressive (we write $p \in \mathcal{R}^{+}$) if it is rd-continuous function and satisfies $1+$ $\mu(t) p(t)>0$ for all $t \in \mathbb{T}$. Hilger in [20] showed that for $p(t) \mathrm{rd}$-continuous and regressive, the solution of the initial value problem

$$
y^{\Delta}(t)=p(t) y(t), \quad y\left(t_{0}\right)=1
$$


is given by the generalized exponential function $e_{p}\left(t, t_{0}\right)$, which is defined by

$$
e_{p}\left(t, t_{0}\right)=\exp \left\{\int_{t_{0}}^{t} \zeta_{\mu(s)}(p(s)) \Delta s\right\}
$$

where $t_{0}, t \in \mathbb{T}$, and the cylinder transformation $\zeta_{h}(z)$ is defined by

$$
\zeta_{h}(z)= \begin{cases}\frac{\log (1+h z)}{h}, & \text { if } h \neq 0, \\ z, & \text { if } h=0,\end{cases}
$$

where $z \in \mathbb{R}$ and $h \in \mathbb{R}^{+}$.

The next lemma can be found in [11].

Lemma 2.1 Assume $t_{0}, t \in \mathbb{T}$.

(i) For a non-negative $\varphi$ with $-\varphi \in \mathcal{R}^{+}$, we have the following inequality:

$$
1-\int_{t_{0}}^{t} \varphi(s) \Delta s \leq e_{-\varphi}\left(t, t_{0}\right) \leq \exp \left\{-\int_{t_{0}}^{t} \varphi(s) \Delta s\right\}
$$

(ii) If $\varphi$ is rd-continuous and non-negative, then

$$
1+\int_{t_{0}}^{t} \varphi(s) \Delta s \leq e_{\varphi}\left(t, t_{0}\right) \leq \exp \left\{\int_{t_{0}}^{t} \varphi(s) \Delta s\right\} .
$$

Lemma 2.2 Assume that $\mathbb{T}$ is a time scale with $t_{0} \in \mathbb{T}$. If $f(t)>0$ on $\left[t_{0}, \infty\right)_{\mathbb{T}}$, then

$$
[\ln f(t)]^{\Delta} \leq \frac{f^{\Delta}(t)}{f(t)}, \quad \text { for } t \in\left[t_{0}, \infty\right)_{\mathbb{T}}
$$

Proof Fix $t$. We consider two cases: (i) $f^{\Delta}(t) \leq 0$ and (ii) $f^{\Delta}(t) \geq 0$.

In the first case, we see that

$$
h \mu(t) f^{\Delta}(t)+f(t) \leq f(t) .
$$

Now recall $f(\sigma(t))=f(t)+\mu(t) f^{\Delta}(t)$ so $h \mu(t) f^{\Delta}(t)+f(t)=h f(\sigma(t))+(1-h) f(t)>0$ and as a result

$$
\frac{1}{h \mu(t) f^{\Delta}(t)+f(t)} \geq \frac{1}{f(t)} .
$$

Apply the chain rule (2.1), and we get (note $\left.f^{\Delta}(t) \leq 0\right)$

$$
[\ln f(t)]^{\Delta}=\left\{\int_{0}^{1} \frac{1}{h \mu(t) f^{\Delta}(t)+f(t)} d h\right\} f^{\Delta}(t) \leq \frac{f^{\Delta}(t)}{f(t)} .
$$

In the second case, we see that

$$
h \mu(t) f^{\Delta}(t)+f(t) \geq f(t) .
$$


Applying the chain rule (2.1), we get

$$
[\ln f(t)]^{\Delta}=\left\{\int_{0}^{1} \frac{1}{h \mu(t) f^{\Delta}(t)+f(t)} d h\right\} f^{\Delta}(t) \leq \frac{f^{\Delta}(t)}{f(t)} .
$$

Thus, we deduce in both cases that

$$
[\ln f(t)]^{\Delta} \leq \frac{f^{\Delta}(t)}{f(t)}
$$

The proof is complete.

Lemma 2.3 Assume that $\mathbb{T}$ is a time scale with $t_{0} \in \mathbb{T}$. If $f(t)>0$ and $f^{\Delta}(t) \geq 0$ for $t \in$ $\left[t_{0}, \infty\right)_{\mathbb{T}}$, then for $\alpha>0$

$$
\left[e^{-\alpha f(t)}\right]^{\Delta} \geq-\alpha e^{-\alpha f(t)} f^{\Delta}(t)
$$

Proof Since $f(t)>0$ and $f^{\Delta}(t) \geq 0$ for $t \in\left[t_{0}, \infty\right)_{\mathbb{T}}$, we have for $h \in(0,1)$

$$
f(t) \leq h \mu(t) f^{\Delta}(t)+f(t)
$$

Applying the chain rule (2.1) and using (2.4), we see that

$$
\left[e^{-\alpha f(t)}\right]^{\Delta}=\left\{-\alpha \int_{0}^{1} e^{-\alpha\left(f(t)+h \mu(t) f^{\Delta}(t)\right)} d h\right\} f^{\Delta}(t) \geq-\alpha e^{-\alpha f(t)} f^{\Delta}(t) .
$$

The proof is complete.

\section{Lower and upper bounds for $x(\tau(t)) / x(t)$}

In this section, we establish lower and upper bounds for $x(\tau(t)) / x(t)$ where $x(t)$ is a solution of equation (1.5). We use the notation $\tau^{0}(t)=t$ and inductively define the iterates of $\tau^{-i}(t)$ by

$$
\tau^{-i}(t)=\left(\tau^{-1} \circ \tau^{-(i-1)}\right)(t), \quad \text { for } i=1,2, \ldots
$$

where $\tau^{-1}(t)$ is the inverse function of $\tau(t)$. From the definition it is clear that

$$
\tau(t)<t<\tau^{-1}(t)<\cdots<\tau^{-(n-1)}(t)<\tau^{-n}(t)<\cdots .
$$

To find the lower bound for $x(\tau(t)) / x(t)$ we define for $0<\rho<1$ a sequence $f_{n}(\rho)$ by

$$
\left\{\begin{array}{l}
f_{0}(\rho)=1, \quad f_{1}(\rho)=1 / \rho, \\
f_{n+2}(\rho)=\frac{f_{n}(\rho)}{f_{n}(\rho)+1-e^{(1-\rho) f_{n}(\rho)}}, \quad n=0,1,2,3, \ldots
\end{array}\right.
$$

We note some properties of $f_{n}(\rho)$ for the reader's interest (see [9] or use an elementary argument using $\left.\frac{x}{x+1-e^{(1-\rho) x}}\right)$. For $0<1-\rho \leq 1 / e$, we have

$$
1 \leq f_{n}(\rho) \leq f_{n+2}(\rho) \leq e, \quad n=0,1,2, \ldots,
$$


so there exists a function $f(\rho)$ such

$$
\lim _{n \rightarrow \infty} f_{n}(\rho)=f(\rho), \quad 1 \leq f(\rho) \leq e,
$$

where $f(\rho)$ satisfies

$$
f(\rho)=e^{(1-\rho) f(\rho)} .
$$

If $(1-\rho)>1 / e$, then either $f_{n}(\rho)$ is nondecreasing and $\lim _{n \rightarrow \infty} f_{n}(\rho)=+\infty$ or $f_{n}(\rho)$ is negative or $f_{n}(\rho)$ is $\infty$ after a finite numbers of terms.

Theorem 3.1 Assume that $\mathbb{T}$ is a time scale and $t^{\prime}, t_{0}, t_{1} \in \mathbb{T}, t_{0} \geq t^{\prime}, t_{1} \geq \tau^{-3}\left(t_{0}\right), x(t)$ is a solution of $(1.5)$ on $\left[t^{\prime}, \infty\right)_{\mathbb{T}}, x(t)$ is positive on $\left[t_{0}, t_{1}\right]_{\mathbb{T}}$ and there exists $\rho \in(0,1)$ with $\infty>f_{n}(\rho)>0$ for $n \in\{2,3, \ldots\}$ and

$$
\sup _{\lambda \in E}\left\{\lambda \exp \left\{\int_{\tau(t)}^{t} \zeta_{\mu(s)}(-\lambda p(s)) \Delta s\right\}\right\} \leq \rho \quad \text { for } t \in\left[\tau^{-3}\left(t_{0}\right), t_{1}\right]_{\mathbb{T}}
$$

here $E=\left\{\lambda: \lambda>0,1-\lambda p(t) \mu(t)>0\right.$ for $\left.t \in\left[\tau^{-2}\left(t_{0}\right), t_{1}\right]_{\mathbb{T}}\right\}$. Then for $n \geq 0$ when $\tau^{-(2+n)}\left(t_{0}\right) \leq$ $t_{1}$ we have

$$
\frac{x(\tau(t))}{x(t)} \geq f_{n}(\rho), \quad \text { for } t \in\left[\tau^{-(2+n)}\left(t_{0}\right), t_{1}\right]_{\mathbb{T}}
$$

where $f_{n}(\rho)$ is defined in (3.1).

Proof From (1.5), we see that

$$
x^{\Delta}(t)=-p(t) x(\tau(t)) \leq 0, \quad \text { for } t \in\left[\tau^{-1}\left(t_{0}\right), t_{1}\right]_{\mathbb{T}}
$$

so since $x(t)$ is nonincreasing on $\left[\tau^{-1}\left(t_{0}\right), t_{1}\right]_{\mathbb{T}}$ we have

$$
\frac{x(\tau(t))}{x(t)} \geq 1, \quad \text { for } t \in\left[\tau^{-2}\left(t_{0}\right), t_{1}\right]_{\mathbb{T}}
$$

Note (3.5) and the fact that $x$ is positive on $\left[t_{0}, t_{1}\right]_{\mathbb{T}}$, so for $t \in\left[\tau^{-2}\left(t_{0}\right), t_{1}\right]_{\mathbb{T}}$ we have (note $x(\sigma(t))>0$ since $\left.\sigma(t) \geq t \geq \tau^{-2}\left(t_{0}\right)>t_{0}\right)$

$$
\begin{aligned}
0 & =-\mu(t)\left[x^{\Delta}(t)+p(t) x(\tau(t))\right] \\
& =x(t)-x(\sigma(t))-\mu(t) p(t) x(\tau(t)) \\
& <x(t)-\mu(t) p(t) x(t) \\
& =[1-\mu(t) p(t)] x(t) .
\end{aligned}
$$

Hence $1-\mu(t) p(t)>0$ for $t \in\left[\tau^{-2}\left(t_{0}\right), t_{1}\right]_{\mathbb{T}}$ so $-p \in \mathcal{R}^{+}$on the interval $\left[\tau^{-2}\left(t_{0}\right), t_{1}\right]_{\mathbb{T}}$. Using Lemma 2.1 (with the time scale $\left[\tau^{-2}\left(t_{0}\right), t_{1}\right]_{\mathbb{T}}$ ) and (3.3), we have for $t \in\left[\tau^{-3}\left(t_{0}\right), t_{1}\right]_{\mathbb{T}}$ (note 


$$
\begin{aligned}
\left.\tau(t) \in\left[\tau^{-2}\left(t_{0}\right), t_{1}\right]_{\mathbb{T}}\right) & \\
\int_{\tau(t)}^{t} p(s) \Delta s & \geq 1-\exp \left\{\int_{\tau(t)}^{t} \zeta_{\mu(s)}(-p(s)) \Delta s\right\} \\
& \geq 1-\sup _{\lambda \in E}\left\{\lambda \exp \left\{\int_{\tau(t)}^{t} \zeta_{\mu(s)}(-\lambda p(s)) \Delta s\right\}\right\} \\
& \geq 1-\rho .
\end{aligned}
$$

Integrating (1.5) from $\tau(t)$ to $t$, we get

$$
x(\tau(t))=x(t)+\int_{\tau(t)}^{t} p(s) x(\tau(s)) \Delta s,
$$

and hence, for $t \in\left[\tau^{-3}\left(t_{0}\right), t_{1}\right]_{\mathbb{T}}$, we get

$$
x(\tau(t))=x(t)+\int_{\tau(t)}^{t} p(s) x(\tau(s)) \Delta s \geq x(t)+x(\tau(t)) \int_{\tau(t)}^{t} p(s) \Delta s \geq x(t)+x(\tau(t))(1-\rho),
$$

so

$$
\frac{x(\tau(t))}{x(t)} \geq \frac{1}{\rho}=f_{1}(\rho)>0, \quad \text { for } t \in\left[\tau^{-3}\left(t_{0}\right), t_{1}\right] .
$$

When $\tau^{-4}\left(t_{0}\right) \leq t_{1}$, note, for $t \in\left[\tau^{-4}\left(t_{0}\right), t_{1}\right]_{\mathbb{T}}$ and $\tau(t) \leq s \leq t$, that

$$
\int_{\tau(s)}^{\tau(t)} \frac{x^{\Delta}(\xi)}{x(\xi)} \Delta \xi+\int_{\tau(s)}^{\tau(t)} p(\xi) \frac{x(\tau(\xi))}{x(\xi)} \Delta \xi=0
$$

so from Lemma 2.2 we have

$$
\int_{\tau(s)}^{\tau(t)}[\ln x(\xi)]^{\Delta} \Delta \xi+\int_{\tau(s)}^{\tau(t)} p(\xi) \frac{x(\tau(\xi))}{x(\xi)} \Delta \xi \leq 0,
$$

which implies that

$$
\frac{x(\tau(s))}{x(\tau(t))} \geq \exp \left\{\int_{\tau(s)}^{\tau(t)} p(\xi) \frac{x(\tau(\xi))}{x(\xi)} \Delta \xi\right\}
$$

and so using (3.5), we have (note $\xi \in\left[\tau^{-2}\left(t_{0}\right), t_{1}\right]_{\mathbb{T}}$ since $\tau(t) \leq s \leq t$ and $t \in\left[\tau^{-4}\left(t_{0}\right), t_{1}\right]_{\mathbb{T}}$ )

$$
\frac{x(\tau(s))}{x(\tau(t))} \geq \exp \left\{f_{0}(\rho) \int_{\tau(s)}^{\tau(t)} p(\xi) \Delta \xi\right\}
$$

we write $f_{0}(\rho)$ (which is of course 1 here) to indicate the general procedure. Now applying Lemma 2.3 and using (3.6), (3.7) and (3.10), we get (here $t \in\left[\tau^{-4}\left(t_{0}\right), t_{1}\right]_{\mathbb{T}}$ )

$$
\begin{aligned}
x(\tau(t)) & =x(t)+\int_{\tau(t)}^{t} p(s) x(\tau(s)) \Delta s \\
& \geq x(t)+x(\tau(t)) \int_{\tau(t)}^{t} p(s) \exp \left\{f_{0}(\rho) \int_{\tau(s)}^{\tau(t)} p(\xi) \Delta \xi\right\} \Delta s
\end{aligned}
$$




$$
\begin{aligned}
& =x(t)+x(\tau(t)) \int_{\tau(t)}^{t} p(s) \exp \left\{f_{0}(\rho)\left(\int_{\tau(s)}^{s} p(\xi) \Delta \xi-\int_{\tau(t)}^{s} p(\xi) \Delta \xi\right)\right\} \Delta s \\
& \geq x(t)+x(\tau(t)) e^{(1-\rho) f_{0}(\rho)} \int_{\tau(t)}^{t} p(s) \exp \left\{-f_{0}(\rho) \int_{\tau(t)}^{s} p(\xi) \Delta \xi\right\} \Delta s \\
& \geq x(t)+x(\tau(t)) e^{(1-\rho) f_{0}(\rho)} \int_{\tau(t)}^{t} \frac{-\left[\exp \left\{-f_{0}(\rho) \int_{\tau(t)}^{s} p(\xi) \Delta \xi\right\}\right]^{\Delta}}{f_{0}(\rho)} \Delta s \\
& =x(t)+x(\tau(t)) e^{(1-\rho) f_{0}(\rho)}\left[\frac{1-\exp \left\{-f_{0}(\rho) \int_{\tau(t)}^{t} p(\xi) \Delta \xi\right\}}{f_{0}(\rho)}\right] \Delta s \\
& \geq x(t)+x(\tau(t))\left(\frac{e^{(1-\rho) f_{0}(\rho)}-1}{f_{0}(\rho)}\right) .
\end{aligned}
$$

Thus, for $t \in\left[\tau^{-4}\left(t_{0}\right), t_{1}\right]_{\mathbb{T}}$, we get

$$
\frac{x(\tau(t))}{x(t)} \geq \frac{f_{0}(\rho)}{f_{0}(\rho)+1-e^{(1-\rho) f_{0}(\rho)}}=f_{2}(\rho)>0 .
$$

Repeating the above procedure, when $\tau^{-(2+n)}\left(t_{0}\right) \leq t_{1}$ we get for $t \in\left[\tau^{-(2+n)}\left(t_{0}\right), t_{1}\right]_{\mathbb{T}}$

$$
\frac{x(\tau(t))}{x(t)} \geq \frac{f_{n-2}(\rho)}{f_{n-2}(\rho)+1-e^{(1-\rho) f_{n-2}(\rho)}}=f_{n}(\rho)>0 .
$$

The proof is complete.

Remark 3.1 From the proof of Theorem 3.1 notice in the statement of Theorem 3.1 we could replace $\infty>f_{n}(\rho)>0$ for $n \in\{2,3, \ldots\}$ with $\infty>f_{n}(\rho)>0$ for $n \in\{2,3, \ldots, N-2\}$ if $\tau^{-(2+N)}\left(t_{0}\right)<t_{1}<\tau^{-(3+N)}\left(t_{0}\right)$ or $\infty>f_{n}(\rho)>0$ for $n \in\{2,3, \ldots, N-3\}$ if $\tau^{-(2+N)}\left(t_{0}\right)=t_{1}<$ $\tau^{-(3+N)}\left(t_{0}\right)$.

To establish the upper bound for $x(\tau(t)) / x(t)$, we define a sequence $g_{m}(\rho)$ by

$$
\left\{\begin{array}{l}
g_{1}(\rho):=\frac{2 \rho}{(1-\rho)^{2}-2 M(1-\rho)}, \\
g_{m+1}(\rho):=\frac{2\left(\rho-\frac{1}{g_{m}(\rho)}\right)}{\left[(1-\rho)^{2}-2 M(1-\rho)\right]},
\end{array}\right.
$$

where $0 \leq \rho<1, m=1,2,3, \ldots$, and $0 \leq M<(1-\rho) / 2$.

We note some properties of $g_{m}(\rho)$ for the reader's interest. Note $g_{m+1}(\rho)<g_{m}(\rho)$, for $m=1,2,3, \ldots$, and trivially

$$
g_{1}(\rho)>\frac{\rho}{(1-\rho)^{2}-2 M(1-\rho)} .
$$

More generally when $0<1-\rho \leq 1 / e$ using an induction argument (i.e. assuming $g_{m}(\rho)>$ $\left.\frac{\rho}{(1-\rho)^{2}-2 M(1-\rho)}\right)$ then

$$
\begin{aligned}
g_{m+1}(\rho) & =\frac{2\left(\rho g_{m}(\rho)-1\right)}{g_{m}(\rho)\left[(1-\rho)^{2}-2 M(1-\rho)\right]} \\
& >\frac{2 \rho}{(1-\rho)^{2}-2 M(1-\rho)}-\frac{2}{\rho}>\frac{\rho}{(1-\rho)^{2}-2 M(1-\rho)}
\end{aligned}
$$


thus $g_{k}(\rho)>\frac{\rho}{(1-\rho)^{2}-2 M(1-\rho)}$ where $k=1,3, \ldots$. Then there exists a function $g(\rho)$ with

$$
\lim _{m \rightarrow \infty} g_{m}(\rho)=g(\rho)=\frac{2}{\rho-\sqrt{2(2 M-1)-4(M-1) \rho-\rho^{2}}} .
$$

for $0<1-\rho \leq 1 / e\left(\right.$ note $2(2 M-1)-4(M-1) \rho-\rho^{2}>0$ if $\left.0<1-\rho \leq 1 / e\right)$.

Theorem 3.2 Assume that $\mathbb{T}$ is a time scale and $t^{\prime}, t_{0} \in \mathbb{T}, t_{0} \geq t^{\prime}, x(t)$ is a solution of $(1.5)$ on $\left[t^{\prime}, \infty\right)_{\mathbb{T}}$, there exists a positive integer $N \geq 4$ such that $x(t)$ is positive on $\left[t_{0}, \tau^{-N}\left(t_{0}\right)\right]_{\mathbb{T}}$ and there exists $\rho \in(0,1)$ with $g_{m}(\rho)>0$ for $m \in\{2,3, \ldots, N-3\}$ and

$$
\sup _{\lambda \in E}\left\{\lambda \exp \left\{\int_{\tau(t)}^{t} \zeta_{\mu(s)}(-\lambda p(s)) \Delta s\right\}\right\} \leq \rho \quad \text { for } t \in\left[\tau^{-3}\left(t_{0}\right), \tau^{-N}\left(t_{0}\right)\right]_{\mathbb{T}}
$$

where $E=\left\{\lambda: \lambda>0,1-\lambda p(t) \mu(t)>0\right.$ for $\left.t \in\left[\tau^{-2}\left(t_{0}\right), \tau^{-N}\left(t_{0}\right)\right]_{\mathbb{T}}\right\}$ and

$$
M=\sup _{s \in\left[\tau^{-3}\left(t_{0}\right), \tau^{-N}\left(t_{0}\right)\right]_{\mathbb{T}}} p(s) \mu(s)<\frac{1-\rho}{2} .
$$

Then for $m \in\{1, \ldots, N-3\}$ we have

$$
\frac{x(\tau(t))}{x(t)}<g_{m}(\rho), \quad \text { for } t \in\left[\tau^{-3}\left(t_{0}\right), \tau^{-(N-m)}\left(t_{0}\right)\right]_{\mathbb{T}}
$$

where $g_{m}(\rho)$ is defined in (3.11).

Proof From (1.5), we see that

$$
x^{\Delta}(t) \leq 0, \quad \text { for } t \in\left[\tau^{-1}\left(t_{0}\right), \tau^{-N}\left(t_{0}\right)\right]_{\mathbb{T}},
$$

and as in Theorem 3.1 notice $1-\mu(t) p(t)>0$ for $t \in\left[\tau^{-2}\left(t_{0}\right), \tau^{-N}\left(t_{0}\right)\right]_{\mathbb{T}}$ so $-p \in \mathcal{R}^{+}$on the interval $\left[\tau^{-2}\left(t_{0}\right), \tau^{-N}\left(t_{0}\right)\right]_{\mathbb{T}}$. From Lemma 2.1 (with the time scale $\left.\left[\tau^{-2}\left(t_{0}\right), \tau^{-N}\left(t_{0}\right)\right]_{\mathbb{T}}\right)$ and (3.12), we have for $t \in\left[\tau^{-3}\left(t_{0}\right), \tau^{-(N-1)}\left(t_{0}\right)\right]_{\mathbb{T}}$ (note $\tau^{-1}(t) \leq \tau^{-N}\left(t_{0}\right)$ )

$$
\int_{\tau(t)}^{t} p(s) \Delta s \geq 1-\rho \quad \text { and } \quad \int_{t}^{\tau^{-1}(t)} p(s) \Delta s \geq 1-\rho .
$$

Let $t \in\left[\tau^{-3}\left(t_{0}\right), \tau^{-(N-1)}\left(t_{0}\right)\right]_{\mathbb{T}}$ and consider

$$
G(r):=\int_{t}^{r} p(s) \Delta s-1+\rho, \quad \text { for } r \in\left[t, \tau^{-1}(t)\right]_{\mathbb{T}} .
$$

Note $G:\left[t, \tau^{-1}(t)\right] \rightarrow \mathbb{R}$ is nondecreasing, $G(t)=-1+\rho<0$, and

$$
G\left(\tau^{-1}(t)\right)=\int_{t}^{\tau^{-1}(t)} p(s) \Delta s-1+\rho \geq 1-\rho-1+\rho=0 .
$$

If $G\left(\tau^{-1}(t)\right)=0$, then

$$
\int_{t}^{\tau^{-1}(t)} p(s) \Delta s=G\left(\tau^{-1}(t)\right)+1-\rho=1-\rho,
$$


whereas if $G\left(\tau^{-1}(t)\right)>0$ then $G(t)<0<G\left(\tau^{-1}(t)\right)$.

In either case (from the intermediate value theorem [20]) there exists $t^{*} \in\left[t, \tau^{-1}(t)\right]_{\mathbb{T}}$ with $\sigma\left(t^{*}\right) \in\left[t, \tau^{-1}(t)\right]_{\mathbb{T}}$ such that $G\left(t^{*}\right) G\left(\sigma\left(t^{*}\right)\right) \leq 0$ and so

$$
\int_{t}^{t^{*}} p(s) \Delta s \leq 1-\rho \quad \text { and } \quad \int_{t}^{\sigma\left(t^{*}\right)} p(s) \Delta s \geq 1-\rho .
$$

Integrating both sides of (1.5) from $t$ to $\sigma\left(t^{*}\right)$, for $t \in\left[\tau^{-3}\left(t_{0}\right), \tau^{-(N-1)}\left(t_{0}\right)\right]_{\mathbb{T}}$, we have

$$
x(t)=x\left(\sigma\left(t^{*}\right)\right)+\int_{t}^{\sigma\left(t^{*}\right)} p(s) x(\tau(s)) \Delta s .
$$

Fix $t \in\left[\tau^{-3}\left(t_{0}\right), \tau^{-(N-1)}\left(t_{0}\right)\right]_{\mathbb{T}}$. Let $s \in \mathbb{T}$ be such that $t \leq s \leq \sigma\left(t^{*}\right) \leq \tau^{-1}(t)$ (here $t^{*}$ is as described above, and note $\tau(t) \leq \tau(s) \leq t$ ) and integrating (1.5) from $\tau(s)$ to $t$ yields

$$
x(\tau(s))=x(t)+\int_{\tau(s)}^{t} p(u) x(\tau(u)) \Delta u
$$

and this together with $x$ being nonincreasing on $\left[\tau^{-1}\left(t_{0}\right), \tau^{-N}\left(t_{0}\right)\right]_{\mathbb{T}}$ and (3.14) will give

$$
\begin{aligned}
x(\tau(s)) & \geq x(t)+x(\tau(t)) \int_{\tau(s)}^{t} p(u) \Delta u \\
& =x(t)+x(\tau(t))\left\{\int_{\tau(s)}^{s} p(u) \Delta u-\int_{t}^{s} p(u) \Delta u\right\} \\
& \geq x(t)+x(\tau(t))\left\{1-\rho-\int_{t}^{s} p(u) \Delta u\right\},
\end{aligned}
$$

so from (3.15), (3.16) and (3.17), we obtain

$$
\begin{aligned}
x(t)= & x\left(\sigma\left(t^{*}\right)\right)+\int_{t}^{\sigma\left(t^{*}\right)} p(s) x(\tau(s)) \Delta s \\
\geq & x\left(\sigma\left(t^{*}\right)\right)+\int_{t}^{\sigma\left(t^{*}\right)} p(s)\left\{x(t)+x(\tau(t))\left\{1-\rho-\int_{t}^{s} p(u) \Delta u\right\}\right\} \Delta s \\
\geq & x\left(\sigma\left(t^{*}\right)\right)+(1-\rho) x(t)+(1-\rho)^{2} x(\tau(t)) \\
& -x(\tau(t))\left\{\int_{t}^{t^{*}} p(s)\left\{\int_{t}^{s} p(u) \Delta u\right\} \Delta s\right. \\
& \left.+\int_{t^{*}}^{\sigma\left(t^{*}\right)} p(s)\left\{\int_{t}^{s} p(u) \Delta u\right\} \Delta s\right\} .
\end{aligned}
$$

Let $F(s)=\int_{t}^{s} p(u) \Delta u$, and note

$$
\begin{aligned}
{\left[F^{2}(s)\right]^{\Delta} } & =2 \int_{0}^{1}\left[h F^{\sigma}(s)+(1-h) F(s)\right] F^{\Delta}(s) d h \\
& =2 \int_{0}^{1}\left[h F^{\sigma}(s)+(1-h) F(s)\right] p(s) d h \\
& \geq 2 F(s) p(s) .
\end{aligned}
$$


Hence,

$$
\begin{aligned}
\int_{t}^{t^{*}} p(s)\left\{\int_{t}^{s} p(u) \Delta u\right\} \Delta s & =\int_{t}^{t^{*}} p(s) F(s) \Delta s \leq \frac{1}{2} F^{2}\left(t^{*}\right) \\
& =\frac{1}{2}\left(\int_{t}^{t^{*}} p(u) \Delta u\right)^{2} \leq \frac{(1-\rho)^{2}}{2}
\end{aligned}
$$

and so we obtain

$$
\begin{aligned}
& \int_{t}^{t^{*}} p(s) \Delta s \int_{t}^{s} p(u) \Delta u+\int_{t^{*}}^{\sigma\left(t^{*}\right)} p(s) \Delta s \int_{t}^{s} p(u) \Delta u \\
& \quad \leq \frac{(1-\rho)^{2}}{2}+\mu\left(t^{*}\right) p\left(t^{*}\right) \int_{t}^{t^{*}} p(u) \Delta u \\
& \quad \leq \frac{(1-\rho)^{2}}{2}+(1-\rho) M .
\end{aligned}
$$

Note $\sigma\left(t^{*}\right) \in\left[t, \tau^{-1}(t)\right]_{\mathbb{T}}, t \in\left[\tau^{-3}\left(t_{0}\right), \tau^{-(N-1)}\left(t_{0}\right)\right]_{\mathbb{T}}$, and $x$ is positive on $\left[t_{0}, \tau^{-N}\left(t_{0}\right)\right]_{\mathbb{T}}$ (so $\left.x\left(\sigma\left(t^{*}\right)\right)>0\right)$. Thus from (3.18) and (3.20), we obtain

$$
\begin{aligned}
x(t) \geq & x\left(\sigma\left(t^{*}\right)\right)+(1-\rho) x(t) \\
& +(1-\rho)^{2} x(\tau(t))-\left[\frac{(1-\rho)^{2}}{2}+(1-\rho) M\right] x(\tau(t)) \\
= & x\left(\sigma\left(t^{*}\right)\right)+(1-\rho) x(t) \\
& +\left[\frac{(1-\rho)^{2}}{2}-(1-\rho) M\right] x(\tau(t))
\end{aligned}
$$

and so we have

$$
\frac{x(\tau(t))}{x(t)}<\frac{2 \rho}{(1-\rho)^{2}-2 M(1-\rho)}=g_{1}(\rho), \quad \text { for } t \in\left[\tau^{-3}\left(t_{0}\right), \tau^{-(N-1)}\left(t_{0}\right)\right]_{\mathbb{T}} .
$$

Fix $t \in\left[\tau^{-3}\left(t_{0}\right), \tau^{-(N-2)}\left(t_{0}\right)\right]_{\mathbb{T}}$ and with $t^{*}$ as described above we have $t \leq \sigma\left(t^{*}\right) \leq \tau^{-1}(t) \leq$ $\tau^{-(N-1)}\left(t_{0}\right)$, so from (3.22) we have

$$
x\left(\sigma\left(t^{*}\right)\right)>\frac{1}{g_{1}(\rho)} x\left(\tau\left(\sigma\left(t^{*}\right)\right)\right)
$$

and since $x$ is nonincreasing on $\left[\tau^{-1}\left(t_{0}\right), \tau^{-N}\left(t_{0}\right)\right]_{\mathbb{T}}$ and $\tau\left(\sigma\left(t^{*}\right)\right) \leq t \leq \tau^{-(N-1)}\left(t_{0}\right)$ we have

$$
x\left(\sigma\left(t^{*}\right)\right)>\frac{1}{g_{1}(\rho)} x\left(\tau\left(\sigma\left(t^{*}\right)\right)\right) \geq \frac{1}{g_{1}(\rho)} x(t) .
$$

Substituting (3.23) into (3.21), we obtain for $t \in\left[\tau^{-3}\left(t_{0}\right), \tau^{-(N-2)}\left(t_{0}\right)\right]_{\mathbb{T}}$ that

$$
x(t)>\frac{1}{g_{1}(\rho)} x(t)+(1-\rho) x(t)+\left[\frac{(1-\rho)^{2}}{2}-(1-\rho) M\right] x(\tau(t)),
$$

and so we have

$$
\frac{x(\tau(t))}{x(t)}<\frac{2\left(\rho-\frac{1}{g_{1}(\rho)}\right)}{(1-\rho)^{2}-2 M(1-\rho)}:=g_{2}(\rho) .
$$


Repeating the above procedure, we obtain for $t \in\left[\tau^{-3}\left(t_{0}\right), \tau^{-(N-m)}\left(t_{0}\right)\right]_{\mathbb{T}}$

$$
\frac{x(\tau(t))}{x(t)}<\frac{2\left(\rho-\frac{1}{g_{m-1}(\rho)}\right)}{(1-\rho)^{2}-2 M(1-\rho)}:=g_{m}(\rho) .
$$

The proof is complete.

\section{Distributions of zeros of solutions}

In this section, we study the distribution of zeros of solutions of (1.5) using the lower and upper bounds for $x(\tau(t)) / x(t)$ in Section 3 .

Theorem 4.1 Assume that $\mathbb{T}$ is a time scale and $t^{\prime}, t_{0} \in \mathbb{T}, t_{0} \geq t^{\prime}, x(t)$ is a solution of $(1.5)$ on $\left[t^{\prime}, \infty\right)_{\mathbb{T}}$, and there exist $\rho \in(0,1)$ and $n_{0}, m_{0} \in\{1,2, \ldots\}$ with $f_{n_{0}}(\rho) \geq g_{m_{0}}(\rho)$, and with

$$
N=2+\min _{n \geq 1, m \geq 1}\left\{n+m: f_{n}(\rho) \geq g_{m}(\rho)\right\}=2+n^{\star}+m^{\star}
$$

assume $\infty>f_{k}(\rho)>0, g_{k}(\rho)>0$ for $n \in\{2,3, \ldots, N-3\}$ and

$$
\sup _{\lambda \in E}\left\{\lambda \exp \left\{\int_{\tau(t)}^{t} \zeta_{\mu(s)}(-\lambda p(s)) \Delta s\right\}\right\} \leq \rho \quad \text { for } t \in\left[\tau^{-3}\left(t_{0}\right), \tau^{-N}\left(t_{0}\right)\right]_{\mathbb{T}}
$$

where $E=\left\{\lambda: \lambda>0,1-\lambda p(t) \mu(t)>0\right.$ for $\left.t \in\left[\tau^{-2}\left(t_{0}\right), \tau^{-N}\left(t_{0}\right)\right]_{\mathbb{T}}\right\}$ and

$$
M=\sup _{s \in\left[\tau^{-3}\left(t_{0}\right), \tau^{-N}\left(t_{0}\right)\right]_{\mathbb{T}}} p(s) \mu(s)<\frac{1-\rho}{2} .
$$

Then every solution of $(1.5)$ cannot be totally positive or totally negative on $\left[t_{0}, \tau^{-N}\left(t_{0}\right)\right]_{\mathbb{T}}$.

Proof Note

$$
f_{n^{\star}}(\rho) \geq g_{m^{\star}}(\rho) \text {. }
$$

Without loss of generality assume $x$ is positive on $\left[t_{0}, \tau^{-N}\left(t_{0}\right)\right]_{\mathbb{T}}$. From Theorem 3.1 we have

$$
\frac{x(\tau(t))}{x(t)} \geq f_{n^{\star}}(\rho), \quad \text { for } t \in\left[\tau^{-\left(2+n^{\star}\right)}\left(t_{0}\right), \tau^{-N}\left(t_{0}\right)\right]_{\mathbb{T}}
$$

and from Theorem 3.2 we have (note $m^{\star}=N-\left(2+n^{\star}\right) \leq N-3$ )

$$
\frac{x(\tau(t))}{x(t)}<g_{m^{\star}}(\rho), \quad \text { for } t \in\left[\tau^{-3}\left(t_{0}\right), \tau^{-\left(N-m^{\star}\right)}\left(t_{0}\right)\right]_{\mathbb{T}^{*}}
$$

Note since $N=2+n^{\star}+m^{\star}$ we have (take $\left.t=\tau^{-\left(N-m^{\star}\right)}\left(t_{0}\right)\right)$

$$
f_{n^{\star}}(\rho) \leq \frac{x\left(\tau^{-\left(1+n^{\star}\right)}\left(t_{0}\right)\right)}{x\left(\tau^{-\left(2+n^{\star}\right)}\left(t_{0}\right)\right)}<g_{m^{\star}}(\rho),
$$

which contradicts (4.1). The proof is complete. 
Theorem 4.2 Assume that $\mathbb{T}$ is a time scale and $t^{\prime}, t_{0} \in \mathbb{T}, t_{0} \geq t^{\prime}, x(t)$ is a solution of $(1.5)$ on $\left[t^{\prime}, \infty\right)_{\mathbb{T}}$, and there exist $\rho \in(0,1)$ and a positive integer $N \geq 4$ and $m_{0} \in\{1,2, \ldots, N-3\}$ with

$$
\int_{\tau\left(t_{m_{0}}\right)}^{t_{m_{0}}} p(s) \Delta s>1-\frac{1}{g_{m_{0}}(\rho)} \quad \text { where } t_{m_{0}}=\tau^{-\left(N-m_{0}\right)}\left(t_{0}\right)
$$

and with

$$
m^{\star}=\min _{m \in\{1, \ldots, N-3\}}\left\{m: \int_{\tau\left(t_{m}\right)}^{t_{m}} p(s) \Delta s>1-\frac{1}{g_{m}(\rho)}\right\} \quad \text { where } t_{m}=\tau^{-(N-m)}\left(t_{0}\right)
$$

assume $\infty>f_{k}(\rho)>0, g_{k}(\rho)>0$ for $n \in\{2,3, \ldots, N-3\}$ and

$$
\sup _{\lambda \in E}\left\{\lambda \exp \left\{\int_{\tau(t)}^{t} \zeta_{\mu(s)}(-\lambda p(s)) \Delta s\right\}\right\} \leq \rho \quad \text { for } t \in\left[\tau^{-3}\left(t_{0}\right), \tau^{-N}\left(t_{0}\right)\right]_{\mathbb{T}},
$$

where $E=\left\{\lambda: \lambda>0,1-\lambda p(t) \mu(t)>0\right.$ for $\left.t \in\left[\tau^{-2}\left(t_{0}\right), \tau^{-N}\left(t_{0}\right)\right]_{\mathbb{T}}\right\}$ and

$$
M=\sup _{s \in\left[\tau^{-3}\left(t_{0}\right), \tau^{-N}\left(t_{0}\right)\right]_{\mathbb{T}}} p(s) \mu(s)<\frac{1-\rho}{2} .
$$

Then every solution of (1.5) cannot be totally positive or totally negative on $\left[t_{0}, \tau^{-N}\left(t_{0}\right)\right]_{\mathbb{T}}$.

Proof Note

$$
\int_{\tau\left(t_{m^{\star}}\right)}^{t_{m^{\star}}} p(s) \Delta s>1-\frac{1}{g_{m^{\star}}(\rho)} \quad \text { where } t_{m^{\star}}=\tau^{-\left(N-m^{\star}\right)}\left(t_{0}\right)
$$

Without loss of generality assume $x$ is positive on $\left[t_{0}, \tau^{-N}\left(t_{0}\right)\right]_{\mathbb{T}}$. From Theorem 3.2 , we have

$$
\frac{x(\tau(t))}{x(t)}<g_{m^{\star}}(\rho), \quad \text { for } t \in\left[\tau^{-3}\left(t_{0}\right), \tau^{-\left(N-m^{\star}\right)}\left(t_{0}\right)\right]_{\mathbb{T}}
$$

so in particular

$$
\frac{x\left(\tau\left(t_{m^{\star}}\right)\right)}{x\left(t_{m^{\star}}\right)}<g_{m^{\star}}(\rho) .
$$

Integrating (1.5) from $\tau\left(t_{m^{\star}}\right)$ to $t_{m^{\star}}$, we obtain

$$
x\left(\tau\left(t_{m^{\star}}\right)\right)-x\left(t_{m^{\star}}\right)=\int_{\tau\left(t_{m^{\star}}\right)}^{t_{m^{\star}}} p(s) x(\tau(s)) \Delta s \geq x\left(\tau\left(t_{m^{\star}}\right)\right) \int_{\tau\left(t_{m^{\star}}\right)}^{t_{m^{\star}}} p(s) \Delta s,
$$

and this together with (4.3) gives

$$
\int_{\tau\left(t_{m^{\star}}\right)}^{t_{m^{\star}}} p(s) \Delta s \leq 1-\frac{x\left(t_{m^{\star}}\right)}{x\left(\tau\left(t_{m^{\star}}\right)\right)} \leq 1-\frac{1}{g_{m^{\star}}(\rho)},
$$

which contradicts (4.2). The proof is complete. 
Theorem 4.3 Assume that $\mathbb{T}$ is a time scale and $t^{\prime}, t_{0} \in \mathbb{T}, t_{0} \geq t^{\prime}, x(t)$ is a solution of $(1.5)$ on $\left[t^{\prime}, \infty\right)_{\mathbb{T}}$, and there exist $\rho \in(0,1)$, a constant $L$ and $n_{0}, m_{0} \in\{1,2, \ldots\}$ with

$$
\frac{1+\ln f_{n_{0}-1}(\rho)}{f_{n_{0}-1}(\rho)}-\frac{1}{g_{m_{0}}(\rho)}<L
$$

and with

$$
N=2+\min _{n \geq 1, m \geq 1}\left\{n+m: L>\left(\frac{1+\ln f_{n-1}(\rho)}{f_{n-1}(\rho)}-\frac{1}{g_{m}(\rho)}\right)\right\}=2+n^{\star}+m^{\star}
$$

assume $\infty>f_{k}(\rho)>0, g_{k}(\rho)>0$ for $n \in\{2,3, \ldots, N-3\}$ and

$$
\sup _{\lambda \in E}\left\{\lambda \exp \left\{\int_{\tau(t)}^{t} \zeta_{\mu(s)}(-\lambda p(s)) \Delta s\right\}\right\} \leq \rho \quad \text { for } t \in\left[\tau^{-3}\left(t_{0}\right), \tau^{-N}\left(t_{0}\right)\right]_{\mathbb{T}}
$$

where $E=\left\{\lambda: \lambda>0,1-\lambda p(t) \mu(t)>0\right.$ for $\left.t \in\left[\tau^{-2}\left(t_{0}\right), \tau^{-N}\left(t_{0}\right)\right]_{\mathbb{T}}\right\}$ and

$$
M=\sup _{\left.s \in\left[\tau^{-3}\left(t_{0}\right) \tau^{-N}\left(t_{0}\right)\right]\right]_{\mathbb{T}}} p(s) \mu(s)<\frac{1-\rho}{2} .
$$

Suppose $f_{n^{\star}-1}(\rho) \geq 1, f_{n^{*}}(\rho)>f_{n^{*}-1}(\rho)$ and for $t^{*} \in\left[\tau\left(t_{1}\right), t_{1}\right]_{\mathbb{T}}\left(\right.$ here $\left.t_{1}=\tau^{-\left(N-m^{\star}\right)}\left(t_{0}\right)\right)$ that

$$
\int_{\tau\left(t_{1}\right)}^{t^{*}} p(s) \Delta s+\int_{\sigma\left(t^{*}\right)}^{t_{1}} p(s) \Delta s \geq L
$$

Then every solution of $(1.5)$ cannot be totally positive or totally negative on $\left[t_{0}, \tau^{-N}\left(t_{0}\right)\right]_{\mathbb{T}}$.

Proof Note

$$
L>\left(\frac{1+\ln f_{n^{*}-1}(\rho)}{f_{n^{*}-1}(\rho)}-\frac{1}{g_{m^{*}}(\rho)}\right) .
$$

Without loss of generality assume $x$ is positive on $\left[t_{0}, \tau^{-N}\left(t_{0}\right)\right]_{\mathbb{T}}$. From Theorem 3.1 , we have

$$
\begin{aligned}
& \frac{x(\tau(t))}{x(t)} \geq f_{n^{*}}(\rho), \quad t \in\left[\tau^{-\left(2+n^{\star}\right)}\left(t_{0}\right), \tau^{-N}\left(t_{0}\right)\right]_{\mathbb{T}}, \\
& \frac{x(\tau(t))}{x(t)} \geq f_{n^{*}-1}(\rho), \quad t \in\left[\tau^{-\left(1+n^{\star}\right)}\left(t_{0}\right), \tau^{-N}\left(t_{0}\right)\right]_{\mathbb{T}}
\end{aligned}
$$

and from Theorem 3.2, we have

$$
\frac{x(\tau(t))}{x(t)}<g_{m^{*}}(\rho), \quad t \in\left[\tau^{-3}\left(t_{0}\right), \tau^{-\left(N-m^{*}\right)}\left(t_{0}\right)\right]_{\mathbb{T}}
$$

so in particular (with $\left.t_{1}=\tau^{-\left(N-m^{\star}\right)}\left(t_{0}\right)=\tau^{-\left(2+n^{\star}\right)}\left(t_{0}\right)\right)$ we have

$$
\frac{x\left(\tau\left(t_{1}\right)\right)}{x\left(t_{1}\right)}<g_{m^{*}}(\rho) .
$$


From (4.6) and $f_{n^{*}}(\rho)>f_{n^{*}-1}(\rho)$ we have

$$
\frac{x\left(\tau\left(t_{1}\right)\right)}{x\left(t_{1}\right)}>f_{n^{*}-1}(\rho) .
$$

Now since $x$ is nonincreasing on $\left[\tau^{-1}\left(t_{0}\right), \tau^{-N}\left(t_{0}\right)\right]_{\mathbb{T}}$ and $f_{n^{\star}-1}(\rho) \geq 1$ (and trivially note $\left.\frac{x\left(\tau\left(t_{1}\right)\right)}{x\left(\tau\left(t_{1}\right)\right)}=1\right)$ there exists a $t^{*} \in\left[\tau\left(t_{1}\right), t_{1}\right]_{\mathbb{T}}$ with

$$
\frac{x\left(\tau\left(t_{1}\right)\right)}{x\left(t^{*}\right)} \leq f_{n^{*}-1}(\rho) \text { and } \quad \frac{x\left(\tau\left(t_{1}\right)\right)}{x\left(\sigma\left(t^{*}\right)\right)} \geq f_{n^{*}-1}(\rho) .
$$

Integrating (1.5) from $\sigma\left(t^{*}\right)$ to $t_{1}$, we obtain

$$
x\left(\sigma\left(t^{*}\right)\right)-x\left(t_{1}\right)=\int_{\sigma\left(t^{*}\right)}^{t_{1}} p(s) x(\tau(s)) \Delta s \geq x\left(\tau\left(t_{1}\right)\right) \int_{\sigma\left(t^{*}\right)}^{t_{1}} p(s) \Delta s,
$$

which implies

$$
\int_{\sigma\left(t^{*}\right)}^{t_{1}} p(s) \Delta s \leq\left(\frac{x\left(\sigma\left(t^{*}\right)\right)}{x\left(\tau\left(t_{1}\right)\right)}-\frac{x\left(t_{1}\right)}{x\left(\tau\left(t_{1}\right)\right)}\right)
$$

From (4.8), (4.9) and (4.10), we obtain

$$
\int_{\sigma\left(t^{*}\right)}^{t_{1}} p(s) \Delta s \leq\left(\frac{1}{f_{n^{*}-1}(\rho)}-\frac{1}{g_{m^{*}}(\rho)}\right)
$$

Divide (1.5) by $x$ and integrate from $\tau\left(t_{1}\right)$ to $t^{*}$, and we get

$$
\int_{\tau\left(t_{1}\right)}^{t^{*}} \frac{x^{\Delta}(s)}{x(s)} \Delta s=-\int_{\tau\left(t_{1}\right)}^{t^{*}} p(s) \frac{x(\tau(s))}{x(s)} \Delta s \leq-f_{n^{*}-1}(\rho) \int_{\tau\left(t_{1}\right)}^{t^{*}} p(s) \Delta s
$$

which implies

$$
\int_{\tau\left(t_{1}\right)}^{t^{*}} p(s) \Delta s \leq-\frac{1}{f_{n^{*}-1}(\rho)} \int_{\tau\left(t_{1}\right)}^{t^{*}} \frac{x^{\Delta}(s)}{x(s)} \Delta s
$$

From (4.9), (4.12) and Lemma 2.2, we obtain

$$
\begin{aligned}
\int_{\tau\left(t_{1}\right)}^{t^{*}} p(s) \Delta s & \leq-\frac{1}{f_{n^{*}-1}(\rho)} \int_{\tau\left(t_{1}\right)}^{t^{*}}[\ln x(s)]^{\Delta} \Delta s=\frac{1}{f_{n^{*}-1}(\rho)} \ln \left(\frac{x\left(\tau\left(t_{1}\right)\right)}{x\left(t^{*}\right)}\right) \\
& \leq \frac{\ln f_{n^{*}-1}(\rho)}{f_{n^{*}-1}(\rho)}
\end{aligned}
$$

and from (4.5), (4.11) and (4.13) we have

$$
\int_{\tau\left(t_{1}\right)}^{t^{*}} p(s) \Delta s+\int_{\sigma\left(t^{*}\right)}^{t_{1}} p(s) \Delta s \leq\left(\frac{1+\ln f_{n^{*}-1}(\rho)}{f_{n^{*}-1}(\rho)}-\frac{1}{g_{m^{*}}(\rho)}\right)<L,
$$

which contradicts (4.4). The proof is complete. 
Remark 4.1 When $\mathbb{T}=\mathbb{R}$ equation (1.5) is the delay differential equation

$$
x^{\prime}(t)+p(t) x(\tau(t))=0, \quad t \in \mathbb{R} .
$$

Theorem 3.1 and Theorem 3.2 are related to the results in [9], Lemma 2.1 and Lemma 2.2, and Theorem 4.3 is motivated from results in [13], Theorem 3.

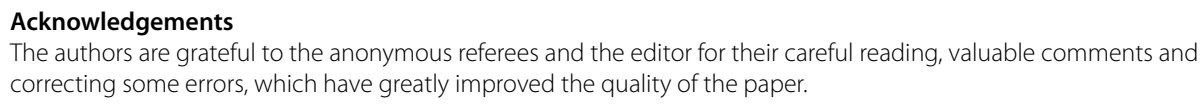

\section{Competing interests}

The authors declare that they have no competing interests.

\section{Authors' contributions}

The authors have contributed equally to this manuscript. They read and approved the final manuscript.

\section{Author details}

${ }^{1}$ School of Mathematics, Statistics and Applied Mathematics, National University of Ireland, Galway, Ireland. ${ }^{2}$ Department of Mathematics, Faculty of Science, Mansoura University, Mansoura, Egypt. ${ }^{3}$ Department of Mathematics, Texas A\&M University, Kingsvilie, TX 78363, USA.

\section{Publisher's Note}

Springer Nature remains neutral with regard to jurisdictional claims in published maps and institutional affiliations.

Received: 26 April 2017 Accepted: 29 June 2017 Published online: 26 July 2017

\section{References}

1. Agarwal, RP, Bohner, M: An oscillation criterion for first order delay dynamic equations. Funct. Differ. Equ. 16, 11-17 (2009)

2. Bohner, M: Some oscillation criteria for first order delay dynamic equations. Far East J. Appl. Math. 18, 289-304 (2005)

3. Bohner, M, Karpuz, B, Ocalan, O: Iterated oscillation criteria for delay dynamic equations of first order. Adv. Differ. Equ. 2008, Article ID 458687 (2008)

4. El-Morshedy, HA: On the distribution of zeros of solutions of first order delay differential equations. Nonlinear Anal. 74, 3353-3362 (2011)

5. Karpuz, B, Ocalan, O: New oscillation tests and some refinements for first-order delay dynamic equations. Turk. J. Math., 1-14 (2015)

6. Liang, FX: The distribution of zeros of solutions of first-order delay differential equations. J. Math. Anal. Appl. 186, 383-392 (1994)

7. Sahiner, Y, Stavroulakis, IP: Oscillations of first order delay dynamic equations. Dyn. Syst. Appl. 15, 645-655 (2006)

8. $\mathrm{Wu}, \mathrm{H}$ : The distribution of zeros of solutions of advanced dynamic equations on time scales. Bull. Malays. Math. Soc. 38, 1-17 (2015)

9. $\mathrm{Wu}, \mathrm{HW}, \mathrm{Xu}, \mathrm{YT}$ : The distribution of zeros of solutions of neutral differential equations. Appl. Math. Comput. 156, 665-677 (2004)

10. Xianhua, T, Jianshe, Y: Distribution of zeros of solutions of first order delay differential equations. Appl. Math. J. Chin. Univ. Ser. B 14, 375-380 (1999)

11. Zhang, BG, Xinghua, D: Oscillation of delay differential equations on time scales. Math. Comput. Model. 36, 1307-1318 (2002)

12. Zhou, Y: The distribution of zeros of solutions of first order functional differential equations. Bull. Aust. Math. Soc. 59, 305-314 (1999)

13. Zhang, BG, Zhou, Y: The distribution of zeros of solutions of differential equations with a variable delay. J. Math. Anal. Appl. 256, 216-228 (2001)

14. Zhou, Y, Zhang, BG: An estimate of numbers of terms of semicycles of delay difference equations. Comput. Math. Appl. 41, 571-578 (2001)

15. Wu, HW, Cheng, SS, Wang, QR: The distribution of zeros of solutions of functional differential equations. Appl. Math. Comput. 193, 154-161 (2007)

16. Tang, $\mathrm{XH}, \mathrm{Yu}, \mathrm{JS}$ : The maximum existence interval of positive solutions of first order delay differential inequalities with applications. Math. Pract. Theory 30, 447-452 (2000)

17. Zhang, BG, Zhou, Y: The semicycles of solutions of delay difference equations. Comput. Math. Appl. 38, 31-38 (1999)

18. Yu, JS, Zhang, BG, Wang, ZC: Oscillation of delay difference equations. Appl. Anal. 53, 117-124 (1994)

19. Zhang, BG, Lian, F: The distribution of generalized zeros of solutions of delay differential equations on time scales. J. Differ. Equ. Appl. 10, 759-771 (2004)

20. Hilger, S: Analysis on measure chains - a unified approach to continuous and discrete calculus. Results Math. 18, 18-56 (1990)

21. Bohner, M, Peterson, A: Dynamic Equations on Time Scales - An Introduction with Applications. Birkhäuser, Boston (2001) 